\title{
Neutron oscillations to parallel world: earlier end to the cosmic ray spectrum?
}

\author{
Zurab Berezhiani ${ }^{1,2, a}$, Askhat Gazizov ${ }^{3, b}$ \\ ${ }^{1}$ Dipartimento di Fisica, Università dell'Aquila, Via Vetoio, 67100 Coppito, L'Aquila, Italy \\ ${ }^{2}$ Laboratori Nazionali Gran Sasso, INFN, 67100 Assergi, L'Aquila, Italy \\ ${ }^{3}$ DESY Zeuthen, Platanenallee 6, 15738 Zeuthen, Germany
}

Received: 5 June 2012 / Revised: 25 July 2012 / Published online: 14 August 2012

(C) The Author(s) 2012. This article is published with open access at Springerlink.com

\begin{abstract}
Present experimental data do not exclude fast oscillation of the neutron $n$ to its degenerate twin from a hypothetical parallel sector, the so called mirror neutron $n^{\prime}$. We show that this effect brings about remarkable modifications of the ultrahigh-energy cosmic ray spectrum testable by the present Pierre Auger Observatory (PAO) and Telescope Array (TA) detector, and the future JEM-EUSO experiment. In particular, the baryon non-conservation during UHECR propagation at large cosmological distances shifts the beginning of the GZK cutoff to lower energies, while in the presence of mirror sources it may enhance the spectrum at $E>100 \mathrm{EeV}$. As a consequence, one can expect a significant reduction of the diffuse cosmogenic neutrino flux.
\end{abstract}

There may exist a hidden parallel sector that is an exact copy of our particle sector. One can imagine a theory based on a direct product $G \times G^{\prime}$ of identical gauge factors with identical particle contents which can emerge, e.g. in the context of $E_{8} \times E_{8}^{\prime}$ string theory. As a minimal possibility, one can consider a case of two Standard Model copies with $G=S U(3) \times S U(2) \times U(1)$ standing for ordinary sector and $G^{\prime}=S U(3)^{\prime} \times S U(2)^{\prime} \times U(1)^{\prime}$ standing for parallel sector. Alternatively, one can envisage some grand unified extensions as $S U(5) \times S U(5)^{\prime}$, etc. The Lagrangians of two worlds can be rendered identical to each other, with all coupling constants being exactly the same in both sectors, by introducing a discrete symmetry $G \leftrightarrow G^{\prime}$ under the exchange of two gauge systems and of the respective matter fields.

A well-known example, coined as mirror world [1-6], was introduced a long time ago for interpreting parity as a discrete symmetry when our 'left-handed' particles are exchanged with their mirror twins that are 'right-handed'.

\footnotetext{
a e-mail: zurab.berezhiani@ aquila.infn.it

be-mail: askhat.gazizov@desy.de
}

Concerns about parity are irrelevant for our following discussions, which can be extended to a parallel sector (or sectors) of any chirality. For us is important only that each ordinary particle: electron $e$, proton $p$, neutron $n$ etc. may have a mass degenerate twin: $e^{\prime}, p^{\prime}, n^{\prime}$ etc. These twin particles must be sterile to our strong and electroweak interactions but have their own strong and electroweak interactions among themselves. ${ }^{1}$

Mirror baryons can be viable as asymmetric dark matter provided that parallel sector has smaller temperature than the ordinary one, $T^{\prime} \ll T$ [7-10]. On the other hand, once this condition is fulfilled, $B-L$ and $C P$ violating interactions among ordinary and mirror particles can generate baryon asymmetries in both sectors [11, 12], naturally giving the relation $\Omega_{\mathrm{B}}^{\prime} / \Omega_{\mathrm{B}} \simeq 5$ between cosmological fractions of the dark and visible matter [13-15]. Such interactions can be mediated by heavy messengers coupled to both sectors, as right handed neutrinos or extra gauge bosons/gauginos [16]. In the context of extra dimensions, ordinary and mirror sectors can be modeled as two parallel 3-dimensional branes and particle processes between them could be mediated by the bulk modes or "baby branes" [17].

The same $B$ or $L$ violating interactions that lead to primordial baryogenesis can also induce mixing phenomena between the ordinary particles and their mirror partners. E.g. effective operator $(1 / M) l \phi l^{\prime} \phi^{\prime}(\Delta L=1)$ between the ordinary/mirror lepton and Higgs fields in the early universe gives rise to an efficient lepto-baryogenesis mechanism for both sectors [11-13], while at low energies it induces the mixing between ordinary (active) neutrinos $\nu_{e, \mu, \tau}$ and their mirror (sterile) partners $v_{e, \mu, \tau}^{\prime}[18,19]$ (see also [20, 21]). ${ }^{2}$

\footnotetext{
${ }^{1}$ In the following, for terminological simplicity, we shall continue to call the particles of the 'primed' parallel sector as mirror particles, independently of their chirality.

${ }^{2}$ Mirror symmetry can be spontaneously broken e.g. due to the difference of weak interaction scales or grand unification scales between two
} 
Effective six-fermion interactions $(1 / M)^{5}(u d d)\left(u^{\prime} d^{\prime} d^{\prime}\right)$, etc. $(\Delta B=1)$ with the scale $M \sim 1-10 \mathrm{TeV}$, involving the ordinary $(u, d)$ and mirror $\left(u^{\prime}, d^{\prime}\right)$ quarks of different families can provide an efficient mechanism for primordial baryogenesis and dark matter genesis, and in addition, can be testable at the LHC [26]. On the other hand, at low energies these operators induce the mass mixing $\varepsilon\left(\bar{n} n^{\prime}+\bar{n}^{\prime} n\right)$ between the neutron $n$ and its mass degenerate mirror twin $n^{\prime}$, with $\varepsilon \sim \Lambda_{\mathrm{OCD}}^{6} / M^{5}$. Hence, in the vacuum conditions $n$ and $n^{\prime}$ must have a maximal mixing while the oscillation time in the rest frame, $\tau_{n n^{\prime}}=\varepsilon^{-1} \sim(M / 10 \mathrm{TeV})^{5} \mathrm{~s}$, can be much smaller than the neutron $\beta$-decay time $\tau_{d} \simeq 880 \mathrm{~s}$, and in fact it can be of the order of a second [26]. This seems rather surprising as it regards the baryon number violating process, but it is not excluded by the present experimental data. ${ }^{3}$ The key moment is that for free neutrons $n-n^{\prime}$ transition is affected by the Earth magnetic field whereas for the neutrons bound in nuclei it is ineffective and so the nuclear destabilization limits are irrelevant. In addition, $n-n^{\prime}$ oscillation with $\tau_{n n^{\prime}} \sim 1 \mathrm{~s}$ is not in conflict with the astrophysical bounds from primordial nucleosynthesis, from the neutron star stability, etc. [26-28]. ${ }^{4}$

In the last years several experiments searched for $n-n^{\prime}$ oscillation via the magnetic field dependence of the neutron losses [32-35]. Note, however, that the lower bounds on $\tau_{n n^{\prime}}$ reported by these experiments (the strongest limit reads $\tau_{n n^{\prime}}>414 \mathrm{~s}$ [34]) and adopted by the Particle Data Group [36] become invalid if the Earth possesses a reasonably large mirror magnetic field: the latter cannot be screened in the experiments and it can drastically influence the probability of $n-n^{\prime}$ oscillation [28]. On the other hand, if dark matter consists of mirror particles, it is also plausible that the solar system and the Earth itself may capture a significant amount of mirror matter due to some feeble interactions between ordinary and mirror particles. The Earth's rotation gives rise to circular currents in the captured mirror matter which may induce a mirror magnetic field up to several Gauss [28].

In the presence of non-zero mirror field $B^{\prime}$ the probability of $n-n^{\prime}$ oscillation has a non-trivial dependence on the ordinary magnetic field $B$ and on its orientation relative to $B^{\prime}$ [28]. In particular, if $B^{\prime}>0.05 \mathrm{G}$, the existing experimental bounds do not exclude $\tau_{n n^{\prime}}<10 \mathrm{~s}$ or so. Moreover, the data acquired in the experiment [35] provide a positive signal for $n-n^{\prime}$ oscillation with $\tau_{n n^{\prime}}$ of few seconds. According to critical analysis performed in Ref. [37],

sectors. Then the mirror sector can be deformed to a shadow world with certain predictable properties. Some phenomenological and cosmological implications of such models were discussed in Refs. [22-25].

${ }^{3}$ Other $\Delta B=1$ effects as e.g. $\Lambda-\Lambda^{\prime}$ oscillation between the hyperons can be even faster. However, they are very short-living and these effects should be more difficult to observe.

${ }^{4}$ For other theoretical work on $n-n^{\prime}$ oscillation see [29-31]. these data indicate that the ultra-cold neutron losses measured in magnetic field depend on the magnetic field strength and its orientation. Namely, the measurements performed at $B=0.2 \mathrm{G}$ show dependence on the magnetic field direction at more than $5 \sigma$ level whereas no effect is seen in the measurements performed at $B=0.4 \mathrm{G}$. This anomaly can be interpreted in terms of $n-n^{\prime}$ oscillation with $\tau_{n n^{\prime}}=2-10 \mathrm{~s}$ provided that the Earth possesses a mirror magnetic field $B^{\prime} \simeq 0.1 \mathrm{G}$ [37]. This result, if confirmed by forthcoming experiments, will have deepest consequences for fundamental particle physics, astrophysics and cosmology.

In this letter we show that fast $n-n^{\prime}$ oscillation can have intriguing implications for the propagation of ultrahighenergy cosmic rays (UHECR). In particular, it may cause significant modifications of the spectrum at $E \gtrsim 10 \mathrm{EeV}$ which can be proved in future experiments with high accuracy.

It is known that the cosmic microwave background (CMB) causes an abrupt end in the cosmic proton spectrum, the so called Greisen-Zatsepin-Kuzmin (GZK) cutoff [38, 39]. The cutoff energy corresponds to the pion photoproduction threshold, $E_{\mathrm{GZK}}=m_{\pi} m / 2 \varepsilon_{\gamma} \simeq 60 \mathrm{EeV}$, where $m$ and $m_{\pi}$ are, respectively, the nucleon and pion masses and $\varepsilon_{\gamma} \simeq 3 T \simeq 10^{-3} \mathrm{eV}$ is an effective energy of relic photons, $T=2.725 \mathrm{~K}$ being the CMB temperature. The mean free path (m.f.p.) of the proton, $l_{s} \sim\left\langle\sigma(p \gamma \rightarrow N \pi) n_{\gamma}\right\rangle^{-1} \propto$ $T^{-3}$, strongly depends on the energy. One has $l_{s} \sim 5 \mathrm{Mpc}$ for $E>300 \mathrm{EeV}$ but it sharply increases at lower energies, e.g. $l_{s}>100 \mathrm{Mpc}$ at $E<60 \mathrm{EeV}$. In each $p \gamma \rightarrow N \pi$ scattering with one pion production the super-GZK protons lose about $15-20 \%$ of their energy, but at large energies, $E \gg E_{\mathrm{GZK}}$, the energy loss by multi-pion production can effectively reach $50 \%$.

The $p \gamma$ scattering has two main pion production channels, $p \gamma \rightarrow p \pi^{0}$ and $p \gamma \rightarrow n \pi^{+}$, with roughly comparable cross sections. Conversion of the cosmic ray proton into the neutron does not influence the propagation length, since $n \gamma \rightarrow N \pi$ scatterings, $n \gamma \rightarrow n \pi^{0}\left(p \pi^{-}\right)$, have nearly the same cross sections as $p \gamma \rightarrow N \pi$ ones. In addition the $\beta$-decay $n \rightarrow p e \tilde{v}_{e}$ converts the neutron back to the proton with practically the same energy. $\mathrm{Up}$ to $E \simeq 0.5 \mathrm{ZeV}$ the decay length $l_{d}=\Gamma c \tau_{d}(\Gamma=E / m$ is Lorentz factor $)$ is smaller than $n \gamma \rightarrow N \pi$ scattering length. Hence, cosmic ray carriers with $E \gg E_{\mathrm{GZK}}$ travel long distances transforming from protons to neutrons and back suffering significant energy losses which downgrade their energy to sub-GZK range. Yet, the baryon number in the cosmic ray propagation is conserved.

In presence of $n-n^{\prime}$ oscillation the situation changes drastically: the produced neutron can now oscillate into a mirror one. If $\tau_{n n^{\prime}} \ll \tau_{d}$ the oscillation length $l_{n n^{\prime}}=c \Gamma \tau_{n n^{\prime}}$ is much smaller than $l_{d}$ and $l_{s}$ so that at these scales oscillations may be averaged. The $n-n^{\prime}$ transition probability was 
calculated in [26, 27] and in general case in [28]. For the cosmic neutron oscillations it reads

$P(E)=\frac{1}{2\left[1+\left(\Gamma \omega \tau_{n n^{\prime}}\right)^{2}\right]}=\frac{1}{2+q(E / 100 \mathrm{EeV})^{2}}$,

where $\omega=\frac{1}{2}\left|\mu_{n} \Delta \mathfrak{B}\right|$ and $\Delta \mathfrak{B}=\mathfrak{B}-\mathfrak{B}^{\prime}, \mu_{n}$ being the neutron magnetic moment and $\mathfrak{B}$ and $\mathfrak{B}^{\prime}$ being, respectively, the ordinary and mirror magnetic fields at the cosmological scales, or more precisely their transverse components. Factor $q=0.45 \times\left(\tau_{n n^{\prime}} / 1 \mathrm{~s}\right)^{2} \times(\Delta \mathfrak{B} / 1 \mathrm{fG})^{2}$, shows the efficiency of $n-n^{\prime}$ oscillation at $E \simeq E_{\mathrm{GZK}}$. Finally, $\beta$-decay of mirror neutron $n^{\prime} \rightarrow p^{\prime} e^{\prime} \tilde{v}_{e}^{\prime}$ converts a cosmic ray, being initially a proton, to a mirror proton.

The latter can be converted to ordinary proton via inverse chain of reactions: $p^{\prime} \gamma^{\prime} \rightarrow n^{\prime} \pi^{\prime}$ decay scattering, $n^{\prime}-n$ transition and $n \rightarrow p e \tilde{v}_{e}$. However, the propagation length of mirror protons is much larger than that of ordinary ones, $l_{s}^{\prime} \gg l_{s}$, since the temperature of mirror CMB is smaller than that of ordinary CMB, $T^{\prime} / T=x \ll 1$. Namely, the Big Bang nucleosynthesis imposes a robust upper bound $x<0.5$ or so [7-9], but the limits strengthen if one assumes that dark matter consists entirely of mirror baryons. In this case the large scale structure and CMB power spectrum require $x<0.3$, while yet stronger limits as $x<0.2$ (or $x<0.1$ ) arise by demanding that Silk damping of mirror baryon perturbations does not prevent the formation of normal (or dwarf) galaxies [7-9, 13].

For the relic mirror photon number density and their average energy we have $n_{\gamma}^{\prime}=x^{3} n_{\gamma}$ and $\varepsilon_{\gamma}^{\prime}=x \varepsilon_{\gamma}$. Thus m.f.p. of mirror cosmic rays is drastically amplified, $l_{s}^{\prime} / l_{s} \simeq x^{-3} \gg$ 1 , while the threshold energy of $p^{\prime} \gamma^{\prime} \rightarrow N^{\prime} \pi^{\prime}$ increases as well, $E_{\mathrm{GZK}}^{\prime} \simeq x^{-1} E_{\mathrm{GZK}}$. So, the energy range $E_{\mathrm{GZK}} \lesssim E \lesssim$ $E_{\mathrm{GZK}}^{\prime}$ acts for ordinary cosmic rays like a sink where they disappear-ordinary cosmic rays with $E>E_{\mathrm{GZK}}$ are converted to mirror ones, but the mirror ones may be converted (at much lower rate) to ordinary ones only at $E>E_{\mathrm{GZK}}^{\prime}$. The dominant fraction of the cosmic rays produced in far distant sources must escape to the parallel sector via $n-n^{\prime}$ oscillation. However, if there are powerful mirror sources the ordinary UHECR flux may be increased at $E>E_{\mathrm{GZK}}^{\prime}$ by the contribution from cosmic rays originated in the mirror sector and converted to ordinary ones via $n^{\prime}-n$ oscillation.

In the presence of $n-n^{\prime}$ oscillation, evolution of the four UHECR number densities $U_{i}=U_{i}(E, t), i=p, n, p^{\prime}, n^{\prime}$, in the expanding universe may be described by a system of coupled integro-differential equations

$$
\begin{aligned}
\frac{\partial U_{i}}{\partial t}= & Q_{i}-3 H(t) U_{i}+\frac{\partial\left[E\left(H(t)+\beta_{i}\right) U_{i}\right]}{\partial E}+\frac{m D_{i j}}{E \tau_{d}} U_{j} \\
& -R_{i}(E, t) U_{i} \\
& +T_{i j}(E) \int_{E}^{\infty} d \tilde{E} W_{j k}(E, \tilde{E}, t) U_{k}(\tilde{E}, t),
\end{aligned}
$$

where $H(t)$ is the Hubble parameter. We assume that cosmic rays sources are distributed homogeneously in space and their generation functions $Q_{i}(E, t)$ may have cosmological evolution with time $t[40,41]$. Here $W_{j k}(E, \tilde{E}, t)$ is the probability density for a nucleon $k\left(N=p, n\right.$ or $\left.N^{\prime}=p^{\prime}, n^{\prime}\right)$ of energy $\tilde{E}$ to transform via the pion-production scatterings off the respective CMB $\left(\gamma\right.$ or $\left.\gamma^{\prime}\right)$ into a nucleon $j$ (again $N$ or $N^{\prime}$ ) with energy $E$. Hence, the mixed terms between two systems vanish, $W_{N N^{\prime}}=0$, whereas the relevant terms are calculated using the cross sections of $p \gamma$ and $n \gamma$ processes which take into account also the multipion production channels. The matrix $T_{i j}(E)$, with $T_{p p}=$ $T_{p^{\prime} p^{\prime}}=1, T_{n n}=T_{n^{\prime} n^{\prime}}=1-P(E), T_{n n^{\prime}}=T_{n^{\prime} n}=P(E)$ given by Eq. (1) and with other elements being zero, stands for transition probabilities due to $n-n^{\prime}$ oscillation: the neutron $n$ produced in $N \gamma$-scattering, $N=p, n$, promptly oscillates into $n^{\prime}$ with a probability $P(E)$ and vice versa; $R_{i}(E, t)=\int_{0}^{E} d \tilde{E} \sum_{j} W_{j i}(\tilde{E}, E, t)$ stands for probability of a nucleon $i$ with energy $E$ to disappear from the energy range $d E$; factors $\beta_{p, p^{\prime}}(E, t)$ take into account the $p$ and $p^{\prime}$ energy losses due to $e^{+} e^{-}$pair production $\left(\beta_{n, n^{\prime}}=0\right)$. The matrix $D_{i j}$ takes into account $n$ and $n^{\prime} \beta$-decays transforming neutrons back into protons with practically the same energy; here $D_{p n}=D_{p^{\prime} n^{\prime}}=1, D_{n n}=D_{n^{\prime} n^{\prime}}=-1$ and all other elements are zero. In the absence of $n-n^{\prime}$ oscillation the system (2) obviously splits into two independent sets of equations for ordinary and mirror cosmic rays.

At all reasonable energies neutrons decay before their $n \gamma \rightarrow N \pi$ scattering off CMB, so that $l_{d}<l_{s} \ll l_{s}^{\prime}$ (needless to say that we take into account also the multi-pion production). The relation $l_{d} \ll l_{s}^{\prime}$ holds very well for mirror neutrons, while for ordinary ones $l_{d}<l_{s}$ is fulfilled only at $E<500 \mathrm{EeV}$. For this energy range the initial proton, after $p \gamma \rightarrow n \pi^{+}$scattering, with following prompt $n-n^{\prime}$ oscillation and neutron decay, instantly transforms into a mirror proton, $p \rightarrow p^{\prime}$ with probability $P(E)$, and vice versa, $p^{\prime} \rightarrow p$, neglecting the propagation periods when a nucleon dwells in the mixed $n-n^{\prime}$ state. This allows to integrate out $n$ and $n^{\prime}$ states and to reduce the system of 4 equations (2) effectively to a system of two equations describing evolution of just $p$ and $p^{\prime}$. It should be noted that this approximation is equivalent also to the approximation when one neglects the difference between $p \gamma$ and $n \gamma$ cross sections.

To facilitate the calculations, the latter system of two integro-differential equations was reduced to an analog of a set of two coupled Fokker-Planck type differential equations by expanding the kernels of integrals in (2) in series at $\tilde{E}=E$ up to second derivatives. This method was proved to be valid by comparison with Monte Carlo simulations in the case of propagation of ordinary protons [42]. Moreover, we assume $q \ll 1$ in Eq. (1), so that $P(E)=1 / 2$. This holds for $\Delta \mathfrak{B} \lesssim 1 \mathrm{fG}$ which is consistent with the limits on extragalactic magnetic fields $\mathfrak{B}>10^{-2}-1 \mathrm{fG}$ given in Ref. [43]. There is no strong evidence for a presence of larger magnetic 
fields in voids. The galactic magnetic field generation mechanism from the density perturbations before the recombination predicts seed magnetic fields at the scales larger than $1 \mathrm{Mpc}$ smaller than $10^{-4}$ fG $[44,45]$. However, the data concerning the intergalactic magnetic fields are controversial and there are some hints that they may be larger than $1 \mathrm{fG}$. Nevertheless, at large scales ordinary and mirror magnetic fields can be strongly correlated, so that their difference $\Delta \mathfrak{B}$ can be small enough [27]. There is also a possibility of the resonance MSW like $n-n^{\prime}$ transitions if magnetic fields larger than $1 \mathrm{fG}$ have turbulent structure at scales less than $1 \mathrm{Mpc}^{5}$

We take into account that intensity of cosmic ray sources may depend on the cosmological redshift $z$ and parametrize the generation functions as

$Q(E) \propto E^{-\gamma_{g}}(1+z)^{m} \Theta\left(z_{\max }-z\right) \Theta\left(E_{\max }-E\right)$,

where $\gamma_{g}$ is a generation spectral index and $m$ is an evolution parameter. We also assume that sources emerge at maximal redshift $z_{\max }$ and their acceleration capacities are limited by energy $E_{\max }$. For the mirror cosmic rays, we assume that their generation function $Q^{\prime}(E)$ has the same shape (3) as that of the ordinary ones but the intensity can be different. In other words, we take the ratio $Q^{\prime} / Q$ to be constant. For the sake of definiteness, in our following computations we take $m=3, z_{\max }=4$ and $E_{\max }=10 \mathrm{ZeV}$. But we would like to stress that our results for the UHECR spectral modification in the relevant energy range practically do not depend on the choice of these parameters.

The results of our calculations for different sets of parameters are shown in Figs. 1 and 2. Let us first discuss the case when there are no mirror cosmic rays sources, $Q^{\prime}=0$. Then cosmic rays with $E>E_{\mathrm{GZK}}$ produced in distant extragalactic sources not only lose their energy during propagation, but also degrade in number owing to $n-n^{\prime}$ transition. This shifts the cutoff in the cosmic ray spectrum to energies lower than $E_{\mathrm{GZK}} \simeq 60 \mathrm{EeV}$. Now the cutoff relates also to the nonconservation of the baryon number: the most part of initial protons with $E>E_{\mathrm{GZK}}$ transforms into mirror protons, thus getting invisible for us, and so the ordinary UHECR flux in the relevant energy range will be reduced with respect to what is expected in the normal GZK scenario; on the other hand, an imaginary mirror observer would detect a flux of mirror UHECR originated in our world. ${ }^{6}$ In other words, the

\footnotetext{
${ }^{5}$ Let us remark that rather large magnetic fields would be necessary for the MSW-like transitions in the bigravity picture when gravity is not fully universal between two sectors, e.g. when ordinary and mirror sectors have separate gravities, forming one massless and one massive graviton states [46, 47].

${ }^{6}$ For demonstration, compare the curves our GZK, our $\rightarrow$ our and our $\rightarrow$ mirror on Fig. 3 which result from real calculations for a concrete choice of parameters $\left(\gamma_{g}=2.4, m=3\right.$ and $\left.T^{\prime} / T=0.3\right)$. Observe that the ordinary sources induce a larger flux of the UHECR in
}

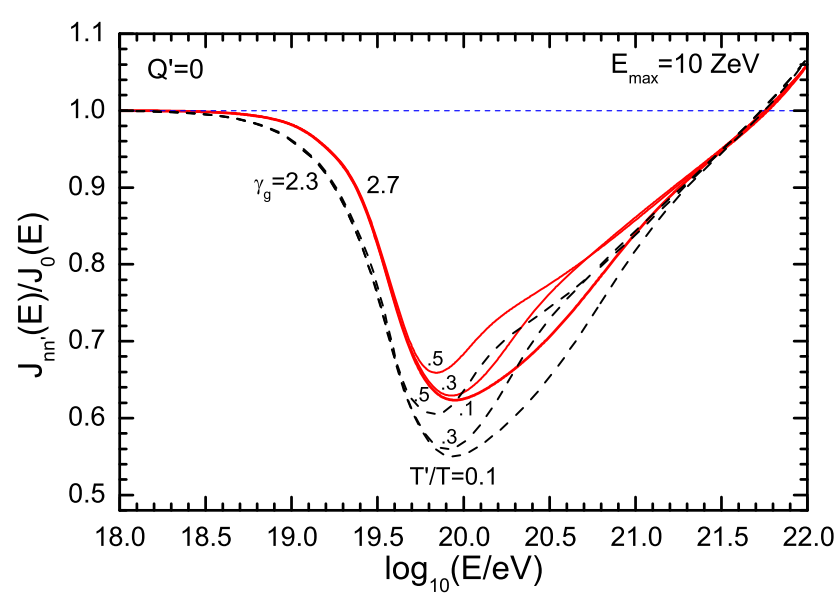

Fig. 1 Ratios of the UHECR spectrum modified by $n-n^{\prime}$ oscillation, $J_{n n^{\prime}}(E)$, and standard GZK (no oscillation) spectrum, $J_{0}(E)$, in the absence of mirror sources, $Q^{\prime}(E)=0$, and for different ratios of CMB temperatures $T^{\prime} / T=0.1,0.3,0.5$. Observe a rather mild dependence on generation spectral indices between $\gamma_{g}=2.3-2.7$

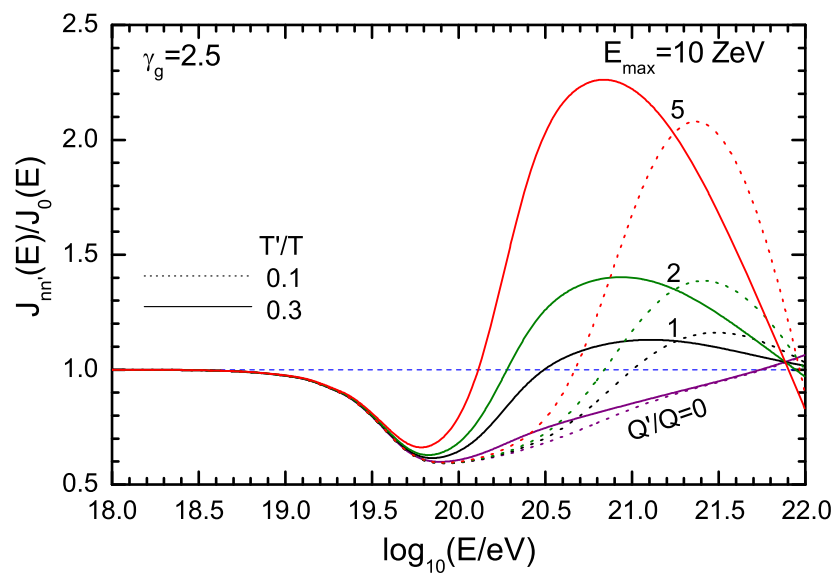

Fig. 2 The same as on Fig. 1 but in the presence of mirror sources with different intensities: $Q^{\prime}(E) / Q(E)=0,1,2,5$. Observe that the modification pattern in the cutoff region is practically independent of the values of $T^{\prime} / T$ and $Q^{\prime} / Q$, but at the energies above $100 \mathrm{EeV}$ the dependence becomes very strong

baryon numbers $B$ and $B^{\prime}$ are not conserved individually, but the sum $B+B^{\prime}$ must be conserved.

In Fig. 1 we show the modification factor $\eta(E)=$ $J_{n n^{\prime}}(E) / J_{0}(E)$, defined as the ratio of UHECR spectra calculated with and without $n-n^{\prime}$ oscillation, in the absence of mirror sources, $Q^{\prime}=0$. Actually, it has an almost universal shape which just weakly depends on the source generation function index $\gamma_{g}$ and on the ratio of CMB temperatures

mirror sector than in our sector, even at energies $E<E_{\mathrm{GZK}}$. For the UHECR produced at high redshift $z$, the $p \gamma \rightarrow N \pi$ reaction threshold decreases by a factor $1+z$ while the respective m.f.p. decreases by a factor $(1+z)^{3}$, whereas for the UHECR that escape to mirror sector the energy losses are much smaller. 
$x=T^{\prime} / T$ in two sectors. The spectral modification due to oscillation starts at $E \sim 10 \mathrm{EeV}$, while the maximal difference from the GZK prediction (about $40 \%$ ) is reached around $E=E_{\mathrm{GZK}}$.

Figure 2 shows the same spectral modification factor $\eta(E)$ in the presence of the mirror sources, for different values of $Q^{\prime}(E) / Q(E)$. In fact, if mirror baryons constitute dark matter, one can expect that there are also mirror cosmic rays. In fact, in quasars and AGN the central black holes can be a potential sites for acceleration of both ordinary and mirror protons. Therefore, it is natural to assume that the ratio $Q^{\prime}(E) / Q(E)$ is the same as the ratio of the relative matter fractions, $\Omega_{B}^{\prime} / \Omega_{B}$. Let us recall that $\Omega_{B}^{\prime} / \Omega_{B} \sim 5$ can be naturally achieved in the joint ordinary-mirror baryogenesis mechanism $[11,12]$ provided that $x<0.3$ or so [13-15].

In this case, in spite of larger m.f.p., the mirror cosmic rays with $E>E_{\mathrm{GZK}}^{\prime}$ can partially move to the ordinary sector which can substantially increase the UHECR flux at energies above $E=100 \mathrm{EeV}$ (compare the curves mirror $\rightarrow$ our and mirror $\rightarrow$ mirror on Fig. 3 which also shows that the GZK threshold energy in mirror world is shifted as $E_{\mathrm{GZK}}^{\prime} / E_{\mathrm{GZK}} \simeq T / T^{\prime}$ ). The value of the turning point depends on the parameter $x=T^{\prime} / T$ as well as on the strength of mirror sources, $Q^{\prime} / Q$. Note, however, that the position of the pre-GZK cutoff remains robust: the shape of the $\eta(E)$ at $E=10-100 \mathrm{EeV}$ practically does not depend on the strength of the mirror sources. ${ }^{7}$

The earlier end of the cosmic ray spectrum seems to be indicated by the data of Pierre Auger Observatory (PAO). The PAO data show the cutoff starting from $E \simeq 25 \mathrm{EeV}$ [48], a factor of 2 lower than $E_{\mathrm{GZK}}$. The estimated systematic uncertainty of $22 \%$ in the energy definition is not sufficient for adjusting the cutoff position to the GZK shape. In Fig. 3 we show the PAO spectrum which is upscaled by $18 \%$ with respect to original data reported in [48]. (This is compatible with the $22 \%$ margins allowed by PAO for systematic errors.) Such upscaling in energies, from the one side, renders the PAO data compatible with the data accumulated by other experiments HiRes [49] and TA [50, 51], in the energy range $E<20 \mathrm{EeV}$ where all experiments reached very good accuracy, and on the other side, with the spectral characteristics predicted by dip model that considers protons as the cosmic ray carriers [40]. One can immediately observe that the standard UHECR spectrum (the red solid curve our GZK on Fig. 3), while is perfectly compatible with the PAO data at $E<20 \mathrm{EeV}$ but gets into evident conflict

\footnotetext{
${ }^{7}$ It is interesting to remark that the fraction of cosmic ray which originates in the parallel sector and then transforms to ordinary cosmic rays due to $n-n^{\prime}$ oscillation, can be composed by anti-protons rather than by protons, depending on the sign of the baryon asymmetry of the parallel world. However, in the high energy cosmic ray showers it is difficult to distinguish experimentally primary protons from antiprotons.
}

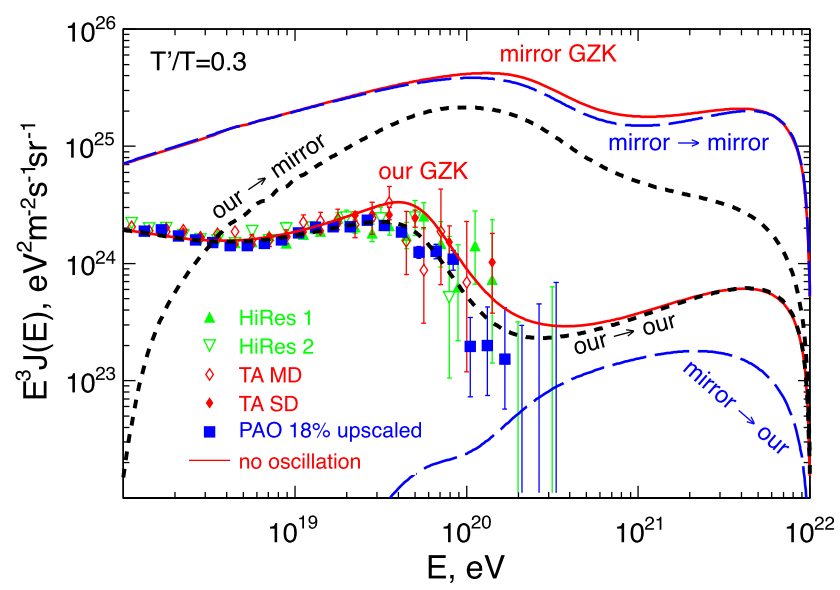

Fig. 3 The red solid curves marked as our GZK and mirror GZK show the expected spectra of the ordinary and mirror cosmic rays in the absence of $n-n^{\prime}$ oscillation. The generation functions in two sectors are taken identical, $Q(E)=Q^{\prime}(E)$, with $\gamma_{g}=2.4$. We also show Experimental results of HiRes, TA and PAO (the latter are $18 \%$ upscaled in energy). The black short-dash curves show the spectra of cosmic rays originated from ordinary sources $Q(E)$ that escape to mirror sector due to $n-n^{\prime}$ oscillation (our $\rightarrow$ mirror) and that remain in our sector (our $\rightarrow$ our $)$. Reciprocally, the blue long-dash curves show mirror and ordinary cosmic ray fluxes originated from mirror sources of the same intensity, $Q^{\prime}(E)=Q(E)$. (Color figure online)

at the energies above $20 \mathrm{EeV} .{ }^{8}$ On the other hand, our result which takes into account $n-n^{\prime}$ oscillation (the black shortdash curve our $\rightarrow$ our on Fig. 3) matches the PAO data much better; in particular, it well reflects the stiffening of the spectrum from $E \simeq 30 \mathrm{EeV}$ or so [48]. As for the HiRes and TA, their data at $E>30 \mathrm{EeV}$ are rather scattered and have large error bars which in fact renders them compatible with the standard GZK prediction as well as to its $n-n^{\prime}$ modification. In fact, the $n-n^{\prime}$ oscillation model proposed in this letter mitigates the controversy between the data of PAO and the data of HiRes and TA experiments putting the beginning of the cutoff in the middle, when extragalactic protons are assumed to be the cosmic ray carriers.

However, we consider that it would be premature to claim that the problem is solved, until the experimental situation is not well settled. A controversy concerning the shape and chemical composition of the UHECR spectrum between the PAO data from one side, and the data of HiRes [49] and TA $[50,51]$ on the other side, is not yet resolved (see e.g. [52]). The HiRes and TA data indicate cosmic rays to be protons with cutoff at $E_{\mathrm{GZK}}$, but their statistic is lower. On the other hand, the PAO data are consistent with protons up to $E \simeq 5-10 \mathrm{EeV}$, but disfavor the protons at larger energies at which the UHECR spectrum seems to be dominated by nuclei. Moreover, it seems that the nuclei must become

\footnotetext{
${ }^{8}$ Needless to say, for the original PAO data [48], without being upscaled by $18 \%$, disagreement with the GZK spectrum is stronger.
} 
gradually heavier with increasing the energy which seems very controversial in itself. It is worth to note, however, that the issue of the composition determining at such high energies is a subject of theoretical models rather than of experimentally measured cross section and multiplicities at the relevant energies. It cannot be excluded that the distribution pattern of the shower maxima observed by the PAO is not due to appearance of the nuclei starting from the energies of about $5 \mathrm{EeV}$ but rather points to new 'strong' physics at the energy scales $\sqrt{s}>10 \mathrm{TeV}$, perhaps the same one which is also at the origin of the baryon number violating interactions as e.g. $(1 / M)^{5}(u d d)\left(u^{\prime} d^{\prime} d^{\prime}\right)$ that generates $n-n^{\prime}$ mixing itself. It should be noted that in case the UHECR are mostly heavy nuclei, the neutrons produced in their photodisintegration on CMB will be also lost as they escape to the parallel world due to $n-n^{\prime}$ oscillation. We think that more data with higher accuracy must be collected by the currently operating PAO and TA experiments for settling the situation which would allow to critically test also our hypothesis.

Prompt neutron oscillations may provide correlation of cosmic rays with $E>100 \mathrm{EeV}$ with distant sources (e.g. BL Lacs $[53,54])$ as an indication of the UHECR transport in the parallel world. It is natural to assume that BL Lacs and blasars in general can be the natural sites for acceleration of both ordinary and mirror cosmic rays, as far as the central black holes considered to be the acceleration engines must be democratic for both types of matter. As far as the CMB of the latter is much colder than ours, the mirror UHECR from the distant sources at several hundred Mpc from us could arrive practically without losing their energy and can be converted to ordinary cosmic rays via $p^{\prime} \gamma^{\prime} \rightarrow n^{\prime} \pi^{\prime}$ scattering within our GZK radius and subsequent conversion $n^{\prime} \rightarrow n$ and decay $n \rightarrow$ pev.

Another immediate consequence of the baryon losses due $n-n^{\prime}$ transitions is a strong suppression of the cosmogenic Berezinsky-Zatsepin (BZ) [55] neutrino flux mostly produced via $p \gamma \rightarrow n \pi^{+}$scattering with $\pi^{+} \rightarrow \mu^{+} v_{\mu}$ and $\mu^{+} \rightarrow e^{+} v_{e} \bar{v}_{\mu}$ decays following. This conclusion remains pessimistic even if ordinary and mirror neutrinos also have non-zero mixing [18, 19]. Really, since $T^{\prime}<T$, mirror cosmic rays have very large m.f.p., $l_{s}^{\prime} \sim x^{-3} l_{s}$ (e.g. $l_{s}^{\prime} \sim$ $600 \mathrm{Mpc}$ for $x=0.2$ versus $l_{s} \sim 5 \mathrm{Mpc}$ ), and thus suffer much less scatterings than ordinary ones. Therefore the diffuse cosmogenic neutrino flux may turn out to be much lower than expected [56]. Even the giant ICECUBE [57] with its control over $1 \mathrm{~km}^{3}$ of ice may be insufficient to detect this flux.

The electromagnetic cascades originated from $p \gamma \rightarrow$ $p \pi^{0}$ channel with subsequent $\pi^{0} \rightarrow 2 \gamma$ decay and $\gamma \gamma_{\mathrm{CMB}}$ $\rightarrow e^{+} e^{-}$will be also suppressed, since most amount of the ordinary super-GZK cosmic rays escape to the parallel world just after few proton scatterings off CMB. In this way, one can soften also the restrictions [56] imposed on the
UHECR models by the diffuse extragalactic gamma-ray flux measured by Fermi-LAT at $E \gtrsim 100 \mathrm{GeV}$ [58].

To conclude, concept of parallel/mirror sector with exactly the same microphysics as the ordinary particle sector provides an interesting possibility for dark matter [7-15] and a peculiar way for its testing via oscillation phenomena of ordinary neutral particles in their mass degenerate mirror twins. In particular, the effects of fast neutron-mirror neutron oscillation may be detected in the laboratory conditions. The present situation is very intriguing in view of the experimental signal for the anomalous neutron losses, which can be explained by $n-n^{\prime}$ oscillation with a timescale of few seconds [37]. If future high accuracy experiments on the neutron disappearance and regeneration will eventually confirm the claim, then deepest consequences will follow for particle physics, astrophysics and cosmology. Namely, the underlying $\mathrm{TeV}$-scale physics can be tested at the LHC, and it can have profound cosmological implications for the primordial baryogenesis and dark matter [26-28]. Here we show that this phenomenon may complementary lead to interesting astrophysical consequences for the cosmic ray spectrum at the GZK region. Due to the baryon non-conservation during the UHECR propagation, the cutoff of the spectrum shifts to lower energies and becomes significantly steeper. Such a spectral modification will be testable at the operating detectors with further increase of the statistics and clearing the controversy regarding the chemical composition. On the other hand, in the presence of powerful mirror sources the UHECR spectrum at highest energies may get even higher than one expects in the standard GZK case. Unfortunately, the data statistics accumulated at the operating installations is not yet enough to determine the exact shape of the spectrum above $100 \mathrm{EeV}$. But this can be achieved at the future JEM-EUSO mission [59].

Acknowledgements The work of Z.B. is partially supported by the MIUR grant PRIN'08 on Astroparticle Physics. A.G. thanks the LNGS for hospitality during the initial period of this work.

Open Access This article is distributed under the terms of the Creative Commons Attribution License which permits any use, distribution, and reproduction in any medium, provided the original author(s) and the source are credited.

\section{References}

1. T.D. Lee, C.N. Yang, Phys. Rev. 104, 254 (1956)

2. I.Yu. Kobzarev, L.B. Okun, Ya.I. Pomeranchuk, Yad. Fiz. 3, 1154 (1966)

3. K. Nishijima, M.H. Saffouri, Phys. Rev. Lett. 14, 205 (1965)

4. S. Blinnikov, M. Khlopov, Sov. Astron. 27, 371 (1983)

5. R. Foot, H. Lew, R.R. Volkas, Phys. Lett. B 272, 67 (1991)

6. H.M. Hodges, Phys. Rev. D 47, 456 (1993)

7. Z. Berezhiani, D. Comelli, F.L. Villante, Phys. Lett. B 503, 362 (2001). arXiv:hep-ph/0008105 
8. A. Ignatiev, R. Volkas, Phys. Rev. D 68, 023518 (2003). arXiv:hep-ph/0304260

9. Z. Berezhiani, P. Ciarcelluti, D. Comelli, F.L. Villante, Int. J. Mod. Phys. D 14, 107 (2005). arXiv:astro-ph/0312605

10. Z. Berezhiani, S. Cassisi, P. Ciarcelluti, A. Pietrinferni, Astropart. Phys. 24, 495 (2006). arXiv:astro-ph/0507153

11. L. Bento, Z. Berezhiani, Phys. Rev. Lett. 87, 231304 (2001). arXiv:hep-ph/0107281

12. L. Bento, Z. Berezhiani, Fortschr. Phys. 50, 489 (2002) arXiv: hep-ph/0111116

13. Z. Berezhiani, Int. J. Mod. Phys. A 19, 3775 (2004). arXiv: hep-ph/0312335

14. Z. Berezhiani, in I. Kogan Memorial Colection, ed. by M. Shifman et al. From Fields to Strings, vol. 3 (2005), pp. 2147-2195. arXiv:hep-ph/0508233

15. Z. Berezhiani, Eur. Phys. J. Spec. Top. 163, 271 (2008). AIP Conf. Proc. 878, 195 (2006). arXiv:hep-ph/0612371

16. Z. Berezhiani, Phys. Lett. B 417, 287 (1998). arXiv:hep-ph/ 9609342

17. G. Dvali, G. Gabadadze, Phys. Lett. B 460, 47 (1999). arXiv: hep-ph/9904221

18. R. Foot, R. Volkas, Phys. Rev. D 52, 6595 (1995). arXiv:hep-ph/ 9505359

19. Z.G. Berezhiani, R.N. Mohapatra, Phys. Rev. D 52, 6607 (1995). arXiv:hep-ph/9505385

20. R. Foot, H. Lew, R.R. Volkas, Mod. Phys. Lett. A 7, 2567 (1992)

21. E.K. Akhmedov, Z. Berezhiani, G. Senjanovic, Phys. Rev. Lett. 69, 3013 (1992). arXiv:hep-ph/9205230

22. Z.G. Berezhiani, A.D. Dolgov, R.N. Mohapatra, Phys. Lett. B 375, 26 (1996). arXiv:hep-ph/9511221

23. Z.G. Berezhiani, Acta Phys. Pol. B 27, 1503 (1996). arXiv: hep-ph/9602326

24. R.N. Mohapatra, V.L. Teplitz, Astrophys. J. 478, 29 (1997). arXiv:astro-ph/9603049

25. C.R. Das, L.V. Laperashvili, H.B. Nielsen, A. Tureanu, Phys. Rev. D 84, 063510 (2011). arXiv:1101.4558 [hep-ph]

26. Z. Berezhiani, L. Bento, Phys. Rev. Lett. 96, 081801 (2006). arXiv:hep-ph/0507031

27. Z. Berezhiani, L. Bento, Phys. Lett. B 635, 253 (2006). arXiv: hep-ph/0602227

28. Z. Berezhiani, Eur. Phys. J. C 64, 421 (2009). arXiv:0804.2088 [hep-ph]

29. Yu.N. Pokotilovski, Phys. Lett. B 639, 214 (2006). arXiv:nucl-ex/ 0601017

30. R.N. Mohapatra, S. Nasri, S. Nussinov, Phys. Lett. B 627, 124 (2005). arXiv:hep-ph/0508109

31. G. Dvali, M. Redi, Phys. Rev. D 80, 055001 (2009). arXiv: 0905.1709 [hep-ph]

32. G. Ban et al., Phys. Rev. Lett. 99, 161603 (2007). arXiv:0705.2336 [nucl-ex]
33. I. Altarev et al., Phys. Rev. D 80, 032003 (2009). arXiv:0905.4208 [nucl-ex]

34. A. Serebrov et al., Phys. Lett. B 663, 181 (2008). arXiv:0706.3600 [nucl-ex]

35. A. Serebrov et al., Nucl. Instrum. Methods Phys. Res., Sect. A, Accel. Spectrom. Detect. Assoc. Equip. 611, 137 (2009). arXiv:0809.4902 [nucl-ex]

36. K. Nakamura et al. (Particle Data Group), J. Phys. G 37, 075021 (2010)

37. Z. Berezhiani, F. Nesti, Eur. Phys. J. C 72, 1974 (2012). arXiv: 1203.1035 [hep-ph]

38. K. Greisen, Phys. Rev. Lett. 16, 748 (1966)

39. G.T. Zatsepin, V.A. Kuzmin, JETP Lett. 4, 78 (1966)

40. V. Berezinsky, A. Gazizov, S. Grigorieva, Phys. Rev. D 74, 043005 (2006). arXiv:hep-ph/0204357

41. V. Berezinsky, A. Gazizov, S. Grigorieva, Phys. Lett. B 612, 147 (2005). arXiv:astro-ph/0502550

42. V. Berezinsky, A. Gazizov, M. Kachelriess, Phys. Rev. Lett. 97, 231101 (2006). arXiv:astro-ph/0612247

43. A.M. Taylor, I. Vovk, A. Neronov, Astron. Astrophys. 529, A144 (2011). arXiv:1101.0932 [astro-ph.HE]

44. Z. Berezhiani, A.D. Dolgov, Astropart. Phys. 21, 59 (2004). arXiv: astro-ph/0305595

45. S. Matarrese, S. Mollerach, A. Notari, A. Riotto, Phys. Rev. D 71, 043502 (2005). arXiv:astro-ph/0410687

46. Z. Berezhiani, F. Nesti, L. Pilo, N. Rossi, J. High Energy Phys. 0907, 083 (2009). arXiv:0902.0144 [hep-th]

47. Z. Berezhiani, L. Pilo, N. Rossi, Eur. Phys. J. C 70, 305 (2010). arXiv:0902.0146 [astro-ph.CO]

48. P. Abreu et al. (Pierre Auger Collaboration). arXiv:1107.4809 [astro-ph.HE]

49. P. Sokolsky (HiRes Collaboration), Nucl. Phys. B, Proc. Suppl. 212-213, 74 (2011)

50. T. Abu-Zayyad et al., arXiv:1202.5141 [astro-ph.IM]

51. T. Abu-Zayyad et al., arXiv:1205.5067 [astro-ph.HE]

52. R. Aloisio, V. Berezinsky, A. Gazizov, Astropart. Phys. 34, 620 (2011). arXiv:1105.1111 [astro-ph.HE]

53. P. Tinyakov, I. Tkachev, JETP Lett. 74, 445 (2001). arXiv: astro-ph/0102476

54. P. Tinyakov, I. Tkachev, Astropart. Phys. 18, 165 (2002). arXiv: astro-ph/0111305

55. V. Berezinsky, G. Zatsepin, Phys. Lett. B 28, 423 (1969)

56. V. Berezinsky, A. Gazizov, M. Kachelriess, S. Ostapchenko, Phys. Lett. B 695, 13 (2011). arXiv:1003.1496 [astro-ph.HE]

57. R. Abbasi et al., Phys. Rev. D 83, 092003 (2011). arXiv: 1103.4250 [astro-ph.CO]

58. A. Abdo et al., Phys. Rev. Lett. 104, 101101 (2010). arXiv: 1002.3603 [astro-ph.HE]

59. Y. Takahashi (JEM-EUSO Collaboration), New J. Phys. 11, 065009 (2009) 